\title{
Breast reduction and appearance
}

B reast reduction is a cosmetic operation because lawsuits that occur after surgery are about the appearance of the breasts, not about a failure to correct the symptoms that lead women to have breast reduction. It should not surprise anyone that the appearance of a woman's breasts after surgery is important. This is not always true, of course. There are women so burdened by large breasts that appearance after surgery is much less important to them than being rid of the weight that they carry daily.

Rapid breast growth to a very large size in a teenage girl can interfere significantly with psychological development, and the decision for or against reduction mammoplasty at a young age can be of Solomonic proportions. This is especially true because the ability to breastfeed can be reduced or eliminated at an age when the decision to do so is almost never 'cool' nor rational, but is certainly always permanent. Of course, reduction mammoplasty is not just cosmetic but is described by everything that plastic surgeons do. It is reconstructive, psychological and cosmetic. Appearance is important in other operations, such as hand surgery and skin cancer surgery. But in reduction mammoplasty, appearance after surgery is especially important and may be the only important thing after surgery because the surgery may be done to relieve symptoms, but if it does not also achieve a nice appearance, the effect of surgery may be lost. Being classified as reconstructive surgery means that all costs related to it are paid by the government and the benefits for this surgery are decided by a government official after viewing submitted forms, symptoms and photographs.

When patients sue after a reduction mammoplasty, the reason is always an unpleasant appearance, never a failure to improve backache nor other symptoms for which the operation received insurance benefits. Therefore, reduction mammoplasty is effective in reducing backache and breast size, and also, reduction mammoplasty is done because women want to improve breast appearance and sue when they conclude that their appearance has not been improved to their satisfaction nor to what they had in mind.

Breast reduction requires a complex consultation, followed by patient-specific notes detailing the problem, choices, pros and cons of various operations, a discussion of general health and risk factors for the operation, and a discussion of the range of results both positive and negative that may result from the procedure. This operation is the lowest remunerated operation in plastic surgery and is either the best deal or the poorest paying surgery, depending on whether you are the patient or surgeon. Our present environment has delinked the ratios between responsibility and reward, except for surgeons who consider these ratios. My concern is that we should relate time and quality of care because there is no doubt that increasing time spent on a surgical problem or on an operation can increase quality. The surgeon, however, should not have to suffer financially for increasing the time spent, especially if it can benefit the patient and the eventual result.

The reduction mammoplasty experience for surgeon and patient perhaps should take longer than it does, considering the implications. Consent needs to cover the likely appearance of the breasts after surgery if everything goes well and especially if it does not. Consent also needs to cover the pros and cons of nipple-areola transfer by pedicle and by free nippleareola graft, which is crucial, as is the possibility of needing to convert one or both pedicles to a free graft(s) due to a poor blood supply after surgery. The necessity to re-operate and the good faith of the patient in allowing the surgeon to do so requires a great trust in the surgeon's judgment expressed immediately after surgery, when there can be a confusion of emotions and even guilt about having the operation done. The issue is tissue death, which is inherent in the operation itself, and the decision to debride and salvage. That I should mention salvage and debridement in an operation where cosmetic appearance after surgery is often paramount, is exactly my point.

Breast reduction in obese patients can seem to be an attractive alternative to those who are frustrated by failing to lose weight. However, this easy fix may be a trap when it fails to produce an ideal breast shape or to result in weight loss. Obese patients can be as interested as anyone else in ideal appearance results, and be unhappy if surgeons assume they have a low body esteem due to their obesity.

Breast reduction, a very common plastic surgery operation, is covered by medicare because of symptoms such as backache, deep bra strap grooves, difficulty in getting clothes that fit and skin rashes under the breasts. An interesting observation is that when legal difficulties occur after reduction mammoplasty, breast appearance is the problem; it is never a failure to relieve the symptoms for which the operation received 
benefits. The consultation and the responsibility for the performance of this operation should be recompensed at a rate in relation to the risk and time spent to ensure that all bases are covered in detail, giving enough time to accommodate this extensive procedure that encompasses every aspect of plastic surgery. This operation is highly cosmetic, meaning the postoperative appearance is important. Because re- muneration reflects risk, time spent and experience, the payment to do breast reduction surgery must reflect all these aspects rather than just the reconstructive aspects of the operation.

John R Taylor jrtaylor@inforamp.net

\section{ANNOUNCEMENT}

\section{Dr A Ross Tilley Plastic and Reconstructive Surgery Foundation Scholarship}

The Dr A Ross Tilley Plastic and Reconstructive Surgery Foundation is now accepting applications for the year 2001

Scholarship.

Dr Tilley was the plastic surgeon who treated burned airmen in the Royal Canadian

Air Force at the Canadian Wing,

East Grinstead, England, during World War II.

He also taught many Canadian plastic surgeons.

Completed applications must be received by March 31, 2001

Applications should be sent to:

Dr John R Taylor

1559 Carletta Drive

Mississauga, Ontario, Canada L4X 1E2

Fax 905-896-7307

E-mail jrtaylor@inforamp.net

\section{1st Annual Toronto Breast Surgery Symposium April 5, 2001 \\ Mount Sinai Hospital, Toronto, Canada}

Program Highlights

Autologous Tissue Breast Reconstruction Oncologic Aspects of Breast Reconstruction

Current Status of Breast Implants

Surgical Approaches in Breast Augmentation

Techniques in Breast Reduction

\section{Faculty}

Wayne Carmen, J Michael Drever, Arnis Freiberg,

Elizabeth Hall-Findlay, Ronald Levine, Hugh McLean, Peter Neligan, Walter Peters

\section{Program Coordinators: \\ Mitchell Brown MD FRCSC \\ John Semple MD FRCSC}

\section{Accreditation}

Royal College/AMA Category 1

For further information please contact:

Continuing Education, Faculty of Medicine University of Toronto, 150 College Street, Room 121

Toronto, Ontario, Canada M5S 3E2

Telephone 416-978-2719

Fax 416-971-2200

E-mail g.jani@utoronto.ca 